\title{
Simulations to Evaluate HIV Vaccine Trial Designs
}

\author{
Andrew L. Adams \\ Department of Epidemiology \\ University of Michigan \\ Daniel C. Barth-Jones \\ Department of Epidemiology \\ University of Michigan
}

\author{
Stephen E. Chick \\ Department of Industrial and Operations Engineering \\ University of Michigan \\ James S. Koopman \\ Department of Epidemiology \\ University of Michigan
}

Many HIV vaccine trials have been proposed to evaluate susceptibility of individuals. However, vaccines may also affect an epidemic's course at the population level by altering the infectiousness of vaccinated individuals who become infected. A vaccine trial design that does not estimate both susceptibility and infectiousness might reject a proposed vaccine that is capable of halting the HIV epidemic. We describe a vaccine trial design called the Retrospective Partner Trial (RPT), which can quantify vaccine effects on both susceptibility and infectiousness. We describe HIVSIM, a simulation environment that generates simulated populations and allows for empirical evaluation of the statistical power of the RPT. HIVSIM explicitly models a number of factors which influence transmission and prevalence, and which have proven difficult to model using standard models. These factors include the infection stage of infected individuals, partnership selection, the duration of partnerships and concurrence, and transmission of HIV. The simulation analysis indicates that the RPT design has substantially greater statistical power for identifying vaccines which, in spite of exhibiting poor protection against infection, are nonetheless capable of halting the HIV epidemic by substantially reducing the infectiousness of vaccinated individuals who become infected.

Keywords: HIV, vaccine, transmission, statistical methods, mathematical models, epidemics, Retrospective Partner Trial, HIVSIM

\section{Background}

The most common vaccine trial design is performed in the same manner as a drug trial. However, it is now understood that important differences in the causal models underlying trials of drugs to cure non-infectious diseases, and vaccines to prevent infectious diseases, cause the drug trial designs to be inadequate for vaccines. This has motivated the development of several new approaches to vaccine trial design [1-10]. This paper describes a new design appropriate for HIV vaccine trials called the Retrospective Partner Trial (RPT) design and illustrates the usefulness of simulations in comparing the power of different trial designs.

To understand how different vaccine trial designs measure different vaccine effects, consider the standard drug trial designs which have commonly been used for vaccine trials [11-17]. In such trials one first enrolls volunteer subjects who are then randomized to receive either the vaccine or a placebo. Neither the subjects, their doctors, nor the investigators know who has received a vaccine and who has received a placebo until the end of the trial. The outcome which is observed in the trial subject is either illness or infection. Subjects might become infected without becoming ill, as is the case with polio.

The underlying causal model for the drug effect analysis is that all of the drug effect is exerted directly upon the drug recipient, and the effect of a drug on one recipient is independent of the effect on other recipients. However, this model is inappropriate for describing vaccines. Vaccines such as the Salk polio 
vaccine alter the course of infection and decrease the frequency of disease associated with infection. By altering the course of infection, they may cause the vaccinated individual who becomes infected to be less infectious to other individuals. Epidemiologists commonly refer to a proportional reduction in susceptibility or infectiousness as the susceptibility or infectiousness effects of the vaccine, respectively. When infection rather than disease is the endpoint used in the drug effect trial design, vaccine infectiousness effects will not be detected. In the case of HIV, a standard trial runs the risk of rejecting vaccines exhibiting poor susceptibility effects, but which could potentially halt the HIV pandemic with their substantial infectiousness effects.

This failure to detect infectiousness effects has not been viewed as a problem until recently. The reasoning was that if a vaccine could not alter the course of infection sufficiently to prevent disease, then it would be unlikely to alter the course enough to reduce infectiousness. This reasoning leads to designs in which the onset of disease is most commonly used as the trial endpoint. However, the typically long times between HIV infection and the onset of AIDS make the onset of disease an impractical endpoint for HIV vaccine trials. Since the biology of HIV infection and vaccines makes the complete prevention of infection problematic, it is important that trials be designed that use infection as a trial endpoint and still detect vaccine effects that reduce infectiousness. The recently published vaccine trial designs that are constructed to detect infectiousness effects [1-8] lack the statistical power to detect those effects when a small number of trial subjects become infected. The RPT design was developed in an attempt to increase statistical power under these circumstances.

There have been several trial designs proposed recently, including those that use partnership information [18]. The RPT design is a further modification of partnership-based designs to estimate vaccine effects on infectiousness. As its name suggests, the fundamental characteristic of this new vaccine trial design is that partners are enrolled retrospectively rather than prospectively. The RPT design was developed in order to make more complete use of information from all available sexual partners when estimating infectiousness effects, thereby increasing the statistical power of the vaccine trial design. The RPT design has two advantages over the aforementioned designs. First, it examines partners in partnerships that were not yet formed when the trial began. Second, it does not examine partners except when the trial participant becomes infected.

Monte Carlo simulation studies have been used to assess the statistical power of some partnership-based designs because of the difficulty in formulating mathematical analyses $[19,20]$. Most of this previous work assumes that transmission events are independent, rather than depending on complex chains of disease transmission. However, Morris and Kretzschmar [43, 44] argue that complex disease dynamics have a significant influence on the conclusions of an epidemiological analysis.

This paper describes the use of HIVSIM, a discreteevent computer simulation (DES), developed for the purpose of quantifying differences between the RPT HIV vaccine trial design and the standard vaccine trial design. Through the retrospective collection of information regarding the timing of sexual partnerships and phylogenetic analysis of HIV viral sequence data, the RPT design estimates the infectiousness effects of the vaccine by comparing the per-partnership transmission probabilities from the infected vaccinated and unvaccinated trial participants.

HIVSIM was developed for the evaluation of the RPT study design to produce simulated vaccine trial data that result from a complex, dynamic HIV transmission model. HIVSIM was constructed as a Monte Carlo DES in a continuous-time framework with randomly determined times to future events. This simulation environment is capable of generating many real-world complexities of trials and trial data which are difficult to generate in simulations based upon continuous compartmental models (e.g., the generation of complex contact patterns between different individuals) [21]. Real-world sexual contact patterns can include partnerships of variable length that might be made preferentially between different types of individuals depending on the partnership status of those individuals. Other characteristics of real world contact patterns include:

1. Concurrent partnerships as well as monogamous partnerships;

2. Partnerships with differing rates of sexual activity which are dependent on both the partners' personal attributes and mutual compatibility of the partners with regard to those attributes; and

3. Individuals with different partnership-seeking propensities based on their intrinsic inclinations for frequency of sexual activity and/or multiplicity of partners.

A DES simulation environment can readily produce all of these real-world contact patterns in a manner that would be impractical with continuous compartmental models.

This paper presents the RPT design and uses the HIVSIM simulations to compare its efficiency to the standard vaccine trial design. An overview of the logical structure of HIVSIM is provided, and then experimental results are presented to demonstrate how data generated by HIVSIM can be used to compare the efficiency of alternative vaccine trial designs. This endeavor provides epidemiologists with an example 
of the utility of the DES simulation framework and its application to the task of epidemiologic study design and evaluation of associated statistical methods.

\section{Evaluation of Vaccine Effects: $V E_{S}, V E_{I}$ and $\mathrm{VE}_{\mathbf{R}}$}

Standard vaccine efficacy estimates measure the vaccine effect on reducing the relative susceptibility of those vaccinated. The vaccine efficacy for susceptibility $\left(\mathrm{VE}_{\mathrm{S}}\right)$ can be estimated as:

$$
V \hat{E}_{S}=1-R R
$$

where $R R$ measures the Relative Risk or Rate of infection in vaccinated as compared to unvaccinated trial participants. Estimation of the relative risk or rate can be made using per-contact transmission probabilities, time-to-infection incidence data or cumulative incidence data [22].

However, the total effectiveness of vaccination depends not only on the reduction of susceptibility in the vaccinated individuals, but also on the reduction of infectiousness in vaccinated individuals who have become infected in spite of vaccination. Estimation of infectiousness effects can only be accomplished by study designs which collect data on the infection of individuals who are exposed to infected vaccine trial participants. In other words, the vaccine effect for infectiousness $\left(V_{\mathrm{I}}\right)$ must be estimated conditional on the exposure to the infected trial subjects who were either vaccine or placebo recipients. $\mathrm{VE}_{\mathrm{I}}$ can be estimated as:

$$
V \hat{E}_{I}=1-\frac{\begin{array}{l}
\text { Fraction of partners who were infected } \\
\text { when exposed to infected placebo recipients }
\end{array}}{\begin{array}{l}
\text { Fraction of partners who were infected } \\
\text { when exposed to infected vaccine recipients }
\end{array}}
$$

The fraction of partners who were infected when exposed to infected trial subjects can be called the secondary attack rate (SAR), and thus we can express the estimate of $\mathrm{VE}_{\mathrm{I}}$ as the following:

$$
V \hat{E}_{I}=1-\frac{S A R_{V}}{S A R_{P}}
$$

The Basic Reproduction Number $\left(\mathbf{R}_{0}\right)$ is a critical measure for the epidemic potential of any infectious disease [23]; and under certain hypothetical conditions, the combined susceptibility and infectiousness vaccination effects can be used to derive a vaccination effect on $R_{0}$. The basic reproduction number is defined as the average number of secondary infections produced when one infected individual is introduced into a population where everyone is susceptible. When $R_{0}$ is greater than one, epidemic disease transmission results; when $R_{0}$ is less than one, disease transmission cannot be sustained and eventually the disease will become eradicated. The vaccine effect on the basic reproduction number can be approximated by:

$$
V \hat{E}_{E}=1-\left(\left(1-V \hat{E}_{S}\right)\left(1-V \hat{E}_{I}\right)\right)
$$

when the following three conditions are met:

- The sexual mixing in a population is random and homogeneous,

- All of the individuals in the population are vaccinated,

- The vaccine does not change the duration of the infection.

$\mathrm{VE}_{\mathrm{R}}$ can be interpreted as the proportional reduction in the basic reproduction number caused by vaccination.

Although these conditions do not hold either in the real world or in our simulations, equation (4) provides a convenient framework for evaluating the maximum potential combined susceptibility and infectiousness effects of vaccines upon transmission in a population. Under these hypothetical conditions, it is possible to derive a critical $V_{R}$ which would be required to reduce the reproduction number for the HIV epidemic in a population to a level below 1 , thus halting the epidemic transmission process. The critical VER under the hypothetical conditions would be given by the equation:

$$
\text { Critical } V E_{R} \geq 1-\frac{1}{R_{0}}
$$

Vaccines which have a $V_{R}$ less than the critical $V_{R}$ could not be expected to halt the epidemic even under the hypothetical conditions used to derive these equations. For example, Blower and McLean estimated the basic reproduction number for the HIV epidemic in San Francisco to be as great as 5.0 [24]. Based on these estimates, only HIV vaccines with a $\mathrm{VE}_{\mathrm{R}}$ of .80 or greater would be capable of halting the epidemic transmission process if the entire population at risk were vaccinated and the other hypothetical conditions were also met.

\section{The RPT Vaccine Trial Design}

The RPT can be distinguished from other proposed vaccine trial designs by the manner in which data to calculate the SARs are collected. The previously proposed designs use prospective follow-up of the partners of vaccine trial subjects to accomplish this. For those trial subjects who have become infected during the vaccine trial, the RPT design uses retrospective contact tracing (also called partner notification) to enroll the sexual partners of the trial subjects in the trial. Two types of information obtainable during the contact tracing process are used to calculate the required SARs:

1. Phylogenetic analysis of blood samples from the trial subjects and their sexual partners, and

2. Timing of the sexual partnerships. 
Phylogenetic analysis is utilized to determine when HIV has been transmitted between those sexual partners [25, 26, 27, 28]. Phylogenetic analysis of all HIV viruses found in blood samples obtained during the trial, and additional HIV samples isolated from the population in the geographic area where the vaccine trial is being conducted, is used to determine if the viruses identified in trial subjects and their partners were likely to have been transmitted between the partners. By considering only HIV viruses that are nearest neighbors on the phylogenetic analysis to be related, the phylogenetic analysis determines if the viruses found in blood samples from two sexual partners were transmitted between those sexual partners.

Using the information from the phylogenetic analysis and the timing of the partnerships, the following five types of sexual partnerships must be successfully distinguished in order to properly calculate the conditional SAR:

1. Partnerships which resulted in the infection of the trial subject,

2. Partnerships which ended prior to the infection of the trial subject,

3. Partnerships with individuals who were already infected with HIV by someone other than the trial subject,

4. Partnerships with uninfected individuals which were in existence after the infection of the trial subject (i.e., partnerships in which transmission was possible but did not occur),
5. Partnerships with infected individuals who were infected by the trial subject.

Proper calculation of an SAR requires the determination of those individuals who could potentially have been infected by the trial subjects (to be included in the denominator of the SAR) and those who actually became infected by the trial subjects (to be included in the numerator of the SAR). Similar to the common epidemiologic procedure of developing rules for the calculation of a household SAR [29], there are various rules by which each partnership might be classified into the preceding five partnership categories or be determined to be unclassifiable. Very conservative rules admit only partnerships where an uninfected partner was very clearly exposed to a study subject in order to estimate the SARs. Less restrictive rules classify a greater number of partnerships as providing relevant information, but run some risk of error. For example, different choices in how to define the time at which a trial subject was infected lead to different rule sets and hence, different implementations of the RPT.

One important advantage of using DES simulation to evaluate the RPT design is the ability to readily evaluate the potential effects of different classification rules on the bias and precision of the vaccine effect estimates produced by the RPT design. This is possible because in the simulated world, one can obtain perfect information. One can know exactly when each individual was infected and by whom and can therefore calculate SARs exactly and establish an upper

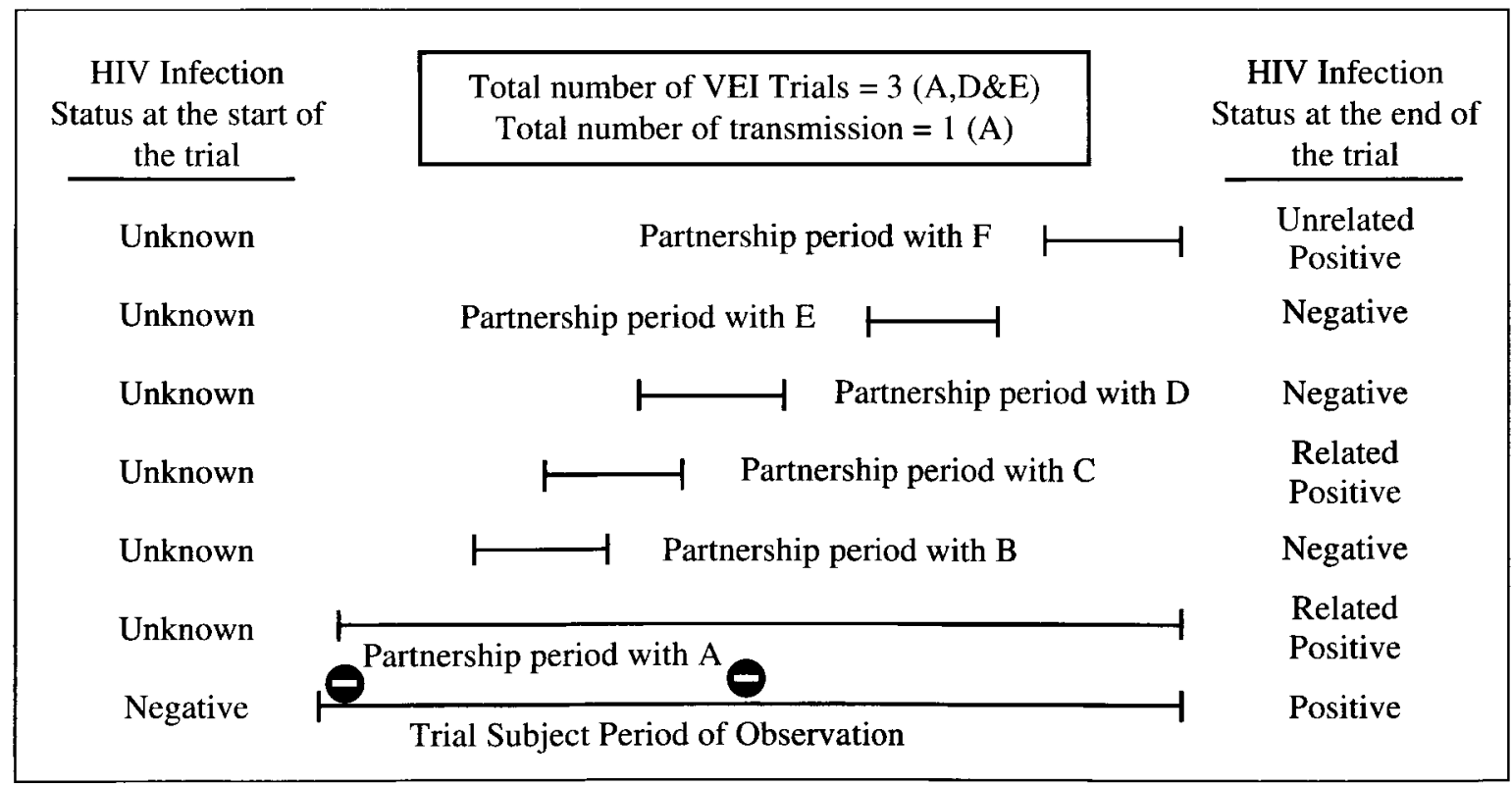

Figure 1. Two hypothetical infection times for the index case are indicated with dots. The inability to determine when the index case became infectious with certainty introduces the potential for bias in the estimates of $V E_{I}$ and $V_{E}$. For example, whether partnership $B$ should have been included in the $S A R$ denominator for $V E_{I}$ is dependent on whether infection of the index cases occurred at the first dot or the second. Evaluation of RPT in a discrete-event simulation environment allows direct comparison between the actual infection times recorded by the simulation model and the infection times chosen by the classification rule logic. 
bound on vaccine effects on infectiousness. In the real world, perfect information is unavailable, but classification rules may provide reasonable estimates of SARs. The evaluation of classification rules is the subject of current research $[30,31]$.

Figure 1 illustrates the timing of six sexual partnerships for one infected trial subject. Time is represented by the horizontal dimension, and the relative timing of the partnerships of the index case is illustrated by the line lengths indicating when each sexual partnership began and ended. The columns at the left- and righthand sides of Figure 1 show obtainable information on the subject's HIV infection status at the start and end, respectively, of the vaccine trial. In addition, the right-hand column indicates whether phylogenetic analysis determined that the HIV sequences in partners were related.

As suggested by the five categories of partnerships enumerated above, an RPT must have a rule that defines the time after which a trial subject case should be considered to have been infectious for HIV. One could define the onset of infectiousness for the trial subject case as the time at which the earliest partnership with related HIV ended. Such a definition accurately reflects the logic that the trial subject case must have been infected by the time the first partnership with related virus ended, but can not accurately determine whether the trial subject case was actually infected at an earlier point in time. Using this logic, the trial subject case in Figure 1 contributes a count of three partnerships to the denominator of the SAR (partnerships A, D and E were in existence after the end of partnership $C$, which was the first partnership to end with a genetically related HIV sequence) and one partnership to the numerator (partnership A was in existence after partnership $C$ ended and thus it is presumed that the trial subject case infected partner A).

Potential biases or loss of precision could result from the inability of classification rules to accurately time the infectiousness of the trial subject case. For example, consider the two hypothetical infection times which are marked with dots in Figure 1. If the first dot on the partnership line for partner $A$ is the actual time of the trial subject's infection, then partnership B should have been included in the SAR denominator.

If the second dot on the partnership line for partner $\mathrm{A}$ is the actual time of the trial subject's infection, then partnership $B$ has been correctly excluded from the SAR denominator.

A classification rule is also needed to treat partnerships involving partners infected with unrelated HIV strains. An example of such a rule is to count all such partners as if they had been infected with HIV prior to forming a partnership with the trial case. This rule thereby classifies the partnerships such as partnership F in Figure 1 as those in which transmission from the index case was not possible and excludes them from the SAR denominator. An important research area for the RPT design is analyzing the effects of these classification rules on the accuracy of the $V_{I}$ and $V E_{R}$ estimates. The evaluation of the RPT design in a DES environment allows direct comparison between the actual infection times as recorded by the simulation model and the infection times decided by the classification rule logic. Another advantage of evaluating the RPT design using a DES is the ability to examine the relationship between various study population characteristics (such as the number of concurrent partnerships found in the study population or the average duration of a sexual partnership) and the accuracy and precision of the $\mathrm{VE}_{\mathrm{I}}$ and $\mathrm{VE}_{\mathrm{R}}$ estimates.

Initial investigations of the RPT design using the HIVSIM model assume that the information obtained from contact tracing and phylogenetic analysis is complete and without error. These assumptions will be relaxed in subsequent investigations in order to examine the effects of missing data, incorrect viral linkages and incorrect timing information which would be present in real-world RPT vaccine trials.

\section{The HIVSIM Simulation Model}

\subsection{The Simulation Model}

The model implemented by HIVSIM is one of an HIV epidemic moving through a closed population of homosexual males. Because the timing, duration and concurrency of partnerships are key factors in the dynamics of the HIV epidemic [32, 33, 34, 43, 44], the simulation explicitly models individuals and the partnerships they form, the sexual contacts that each partnership witnesses, the insertive and receptive role that each partner plays during a contact, and the partnership termination. Individuals seek out new sexual partners at random times and select their partners according to their own characteristics and the characteristics of potential sexual partners. This partnership selection process creates a complex stochastic mixing mechanism dependent on the sexual preferences, concurrency potential and sexual activity frequency of the population.

During a partnership, episodes of sexual contact occur at random intervals, and if one of the partners is infected, each sexual contact presents the opportunity for disease transmission. Consistent with the available epidemiological evidence, the probability of HIV transmission between partners is dependent on the particular sexual activity (oral sex or anal sex), the activity roles assumed by the partners (insertive or receptive) and the stage of the infection in the infected partner $[35,36]$. The natural history of HIV infection is modeled in three stages: primary infection, clinically latent infection and the ARC/AIDS stage [37,38]. Individuals leave the population only by death from AIDS. Finally, a vaccination process is modeled whereby vaccine effects are modeled as proportional reductions in an individual's susceptibility, infectiousness or both. 
HIVSIM can produce a wide variety of sexual contact patterns which impact both HIV transmission dynamics and the potential for the RPT to accurately estimate SARs. The simulation allows the investigator to specify various mixtures of individuals by sex act preferences $[39,40]$, sexual role preferences [41, 42], concurrent partnership potential $[43,44]$, and rates of sexual activity $[45,46]$. It also allows the investigator to specify biases with regard to what kinds of individuals are likely to be linked in partnerships $[47,48$, $49,50]$.

The rest of this section describes in detail the model implemented by HIVSIM. The model is perhaps best understood in terms of the processes in which individuals participate-namely, partnership formation, sexual contact and disease transmission, vaccination, and vaccine trial enrollment. The following sections treat each of these in turn.

\subsection{Partnership Formation}

The partnership formation process utilized by HIVSIM is illustrated by the flow diagram in Figure 2. At initialization, individuals are randomly assigned several attributes that determine partnership formation dynamics and that remain fixed over time. First, an individual is randomly assigned a preference for either anal sex or oral sex. If anal sex is preferred, then the individual is also randomly assigned a preference for either the insertive role or the receptive role during the act. Second, each individual is randomly assigned a limit on the number of concurrent partnerships in which he can participate. This limit is between one and five, inclusive. Finally, each individual is randomly marked as desiring either a high or low sexual contact rate.

At run time, individuals attempt to seek out new partners on dates that are randomly sampled from a geometric distribution whose parameter depends upon their current number of partnerships and whether they have a high or low sexual contact rate.

When an individual attempts to seek out a new partner, a candidate is uniformly sampled from the pool of individuals who currently have less than their maximum number of concurrent partnerships. Once a candidate is chosen, there are three opportunities for this seeker to fail in his attempt to acquire a new partner. First, the candidate has the opportunity to decline the offered partnership. The probability of doing so is conditional upon the difference between the number of partnerships in which the candidate is currently participating and the maximum number in which they are allowed to participate. Second, the possibility exists for the seeker and candidate to decide that their preferred sexual activities and roles render them incompatible. Note that this does not imply that partnerships never form between, for example, two individuals who each prefer the insertive role during anal sex. Rather, there is a probability distribution over the event space $\{$ Anal Insertive, Anal Receptive, Oral $\} \times$ $\{$ Anal Insertive, Anal Receptive, Oral \} that determines the frequency with which individuals with given preferences abort the partnership formation process. Finally, the seeker and candidate may decide that their sexual activity rates render them incompatible. Again, a distribution over the event $\{$ High Rate, Low Rate $\times$ \{High Rate, Low Rate\} determines the frequency with which individuals with given activity rates abort the partnership formation process.

If it is determined that a partnership will not be formed between the seeker and the candidate, then a new interval, $d$, is sampled for the seeker who must then wait $d$ days until next opportunity to acquire a new partner. On the other hand, if it is determined that a partnership will form, then the duration of the partnership is chosen. Partnership durations are a multiple of a sample from one of five randomly chosen Erlang distributions having a shape parameter of 2 and means 2, 6, 30, 180 and 800 days. The probability of choosing a particular distribution is conditional upon the maximum number of concurrent partnerships permitted each partner. The sample multiplier is one of three constants corresponding to the cases when both partners have high rates of sexual activity, when both have low rates of sexual activity, or when the partners have dissimilar rates of sexual activity.

When a given partnership terminates, the intervals during which each partner must wait before next attempting to acquire a new partner are re-sampled in order to reflect the loss of a concurrent partnership. Partnerships may terminate as the result of a scheduled termination event or as the result of the death of a partner.

\subsection{Sexual Episodes, Contacts and HIV Transmission}

Figure 3 illustrates the scheduling of sexual activity and the conditional HIV transmission process within the partnerships simulated by HIVSIM. A sexual episode occurs as soon as a partnership forms. At the end of each episode, the number of days until the next episode is determined and is a multiple of a sample from a geometric distribution whose parameter depends on each partner's preferred sexual activity. The multipliers correspond to the cases when both partners have high rates of sexual contact, when both have low rates of sexual contact and when they have dissimilar rates of sexual contact. The means used in the geometric distributions as well as the aforementioned multipliers are parameters of the model that permit sexual activity rates to be biased as a function of the "compatibility" of the partners' preferences.

Partnerships involve one or more sexual episodes, depending on their duration, and during each episode, partners have exactly one or two sexual contacts. The type (anal or oral) of the first contact as well 
Initialization:

Seek times scheduled

for all individuals

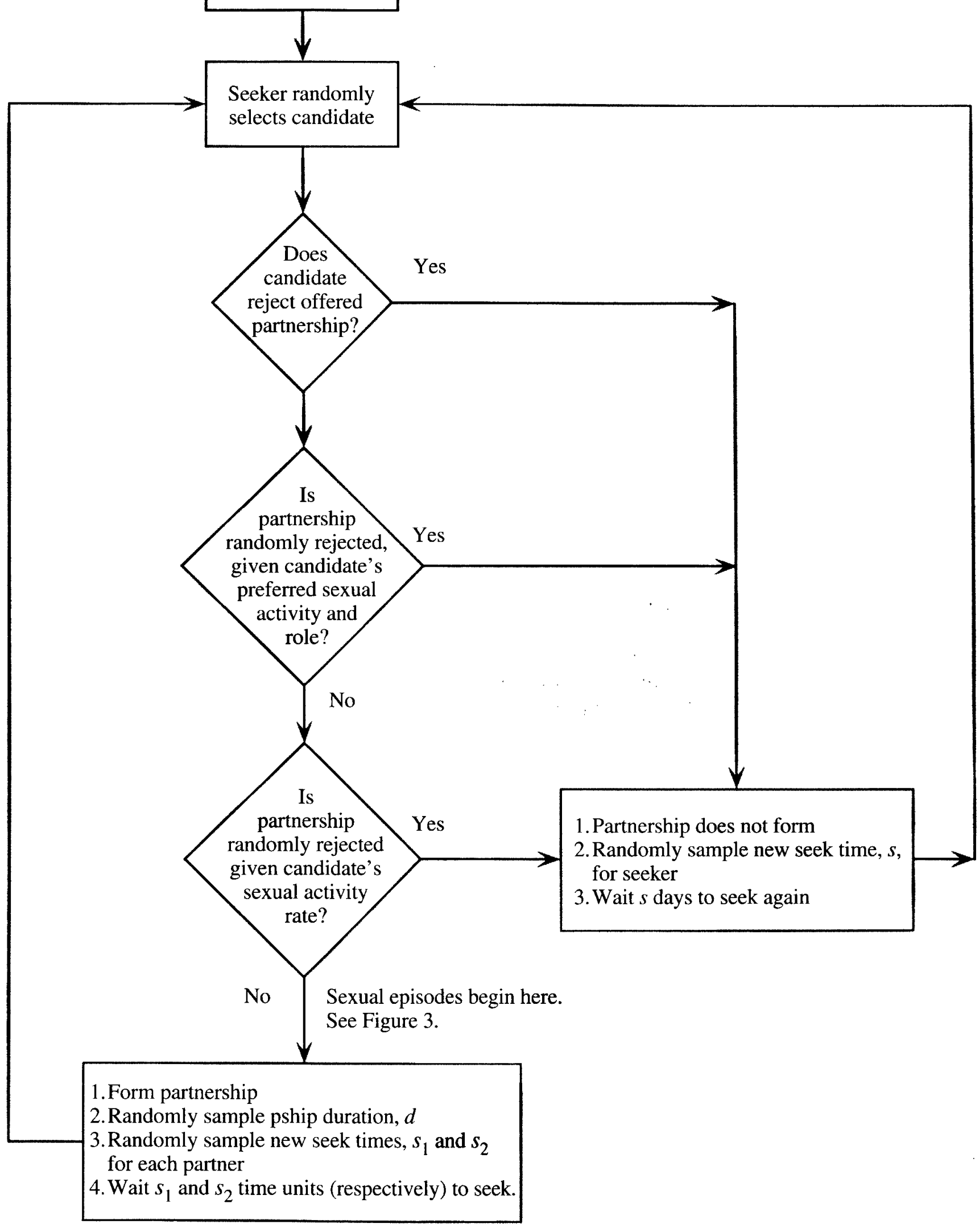

Figure 2. The partnership formation process utilized by HIVSIM. Individuals in the population who have not reached their personal maximum number of concurrent partners are scheduled to randomly seek a candidate partner from the simulated mixing population. The potential partnership may be rejected on the basis of the candidate's concurrency potential, the sexual preference and role compatibility of the seeker and the candidate, or the sexual activity compatibility of the seeker and the candidate. The randomly assigned duration of the partnership, if formed, can be made dependent on the partners' concurrency potentials and sexual activity traits. 


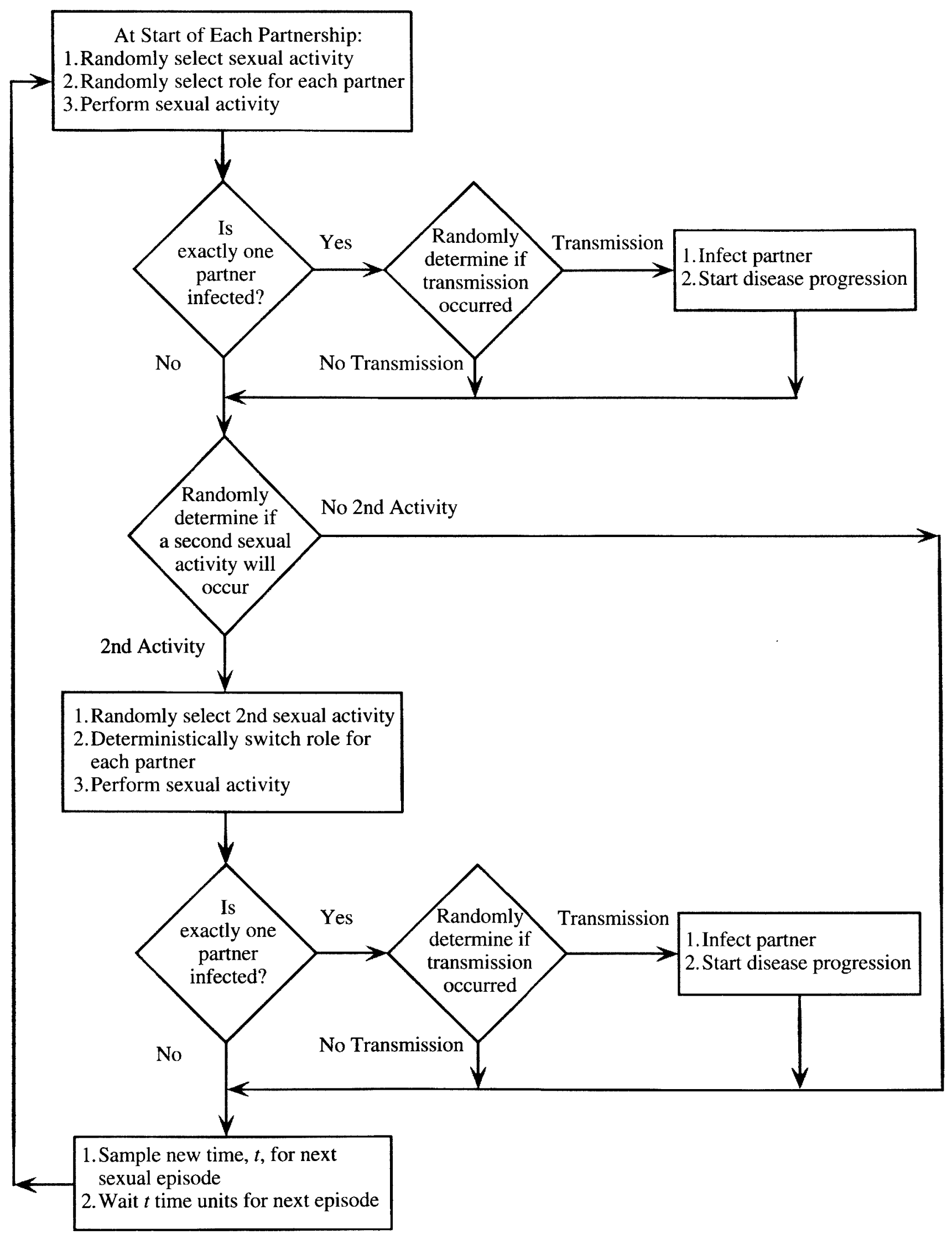

Figure 3. The sexual activity and HIV transmission process utilized by HIVSIM. 
as the role (insertive or receptive) assumed by each partner during the contact are randomly determined and are conditional upon the combination of each partner's preferred sexual activity and sexual role. A second contact occurs with a probability that is constant over all time and all partnerships. If it is determined that a second contact will occur, then the type of contact is randomly determined in the same way as was done for the first contact. However, the roles each partner assumes in the second contact are deterministically exchanged with the roles they assumed in the first contact.

If exactly one of the partners in a given partnership is infected with HIV, then the opportunity for transmission exists during each sexual contact. Consistent with the epidemiological evidence, the probability that an infected individual transmits the infection is dependent upon:

1. The sexual activity in which they are engaged,

2. The role assumed during that activity, and

3. The time since his own infection.

The model assumes a single strain of HIV. The natural history of HIV infection is modeled as a three-stage process wherein infectiousness is highest during the first stage, least in the clinically latent stage and intermediate during the final stage that ends in death due to AIDS-Related Complications. The lengths of stages one, two and three in days are randomly sampled from Erlang distributions with a shape parameter of two and with means of two months, eight years and two years, respectively [37].

\subsection{Vaccination}

The simulation model provides a mechanism by which both the contagiousness and susceptibility effects of a vaccine can independently influence HIV transmission probabilities in order to allow assessments of vaccine efficacy statistics. This mechanism functions as follows. When calculating the transmission probability during a sexual contact as is described in Section 4.3, if the infected partner has been vaccinated, then the probability is multiplied by $1-V E_{I}$. If the uninfected partner has been vaccinated, then the transmission probability is multiplied by $1-V E_{S}$. If both partners have been vaccinated, then the transmission probability is multiplied by both $1-V E_{I}$ and $1-V E_{S}$. Each vaccinated individual's values for $\mathrm{VE}_{\mathrm{S}}$ and $\mathrm{VE}_{\mathrm{I}}$ can be randomly determined, thereby modeling the variability of vaccine effects among individuals. HIVSIM is capable of generating heterogeneous vaccine effects for both $V E_{S}$ and $\mathrm{VE}_{\mathrm{I}}$ in a manner consistent with a frailty mixture model, combining the possibility of complete vaccine failure in some proportion of vaccinated individuals and a Beta distribution of partial effect in the remainder [51, 52]. In a fraction of the study population, the vaccine has no effect and thus $V E_{S}=V E_{I}=0$. In the remaining fraction of the study population, $\mathrm{VE}_{\mathrm{S}}$ and $\mathrm{VE}_{\mathrm{I}}$ are sampled from a Beta distribution.

\subsection{Vaccine Trial Enrollment}

In order to assess statistical measures of vaccine efficacy, the model must contain the notion of a vaccine trial. The model implemented by HIVSIM allows one to specify the desired size and "profile" of the study group, the fraction of the group that is vaccinated and the time at which the study begins (and vaccination occurs). The profile of the group is specified in terms of the fraction of the group having a high or low rate of sexual contact for each of the possible values of maximum concurrent partnerships. If an insufficient number of individuals exist to satisfy a given criterion, then individuals are randomly sampled from the population until the desired group size is achieved. The ability to preferentially recruit members into the vaccine trials who have specific traits regarding sexual activity rates and/or partnership concurrency potential was included in order to allow future investigations of optimal study group profiles.

It should be noted that it is assumed that each trial participant provides complete and accurate partnership information and that all partners can be located. In the real world this may not be the case. One way to evaluate the effect of imperfect partnership information on the RPT design would be to selectively censor partnership information. The current set of simulation runs and the analysis presented here only provide an upper bound on the statistical power of the RPT design.

\subsection{Implementation Details}

HIVSIM is written in $\mathrm{C}_{+}+$and builds upon the SIMEX simulation library produced by the National Micropopulation Simulation Resource Center at the University of Minnesota. SIMEX provides a number of $\mathrm{C}++$ classes to aid in the construction of discrete-event simulations, such as those for event queue management, random number generation and data acquisition. Simulations were run on Hewlett-Packard workstations running the HP/UX operating system; however, the simulation code has been ported to other variants of UNIX, including those that run on PC architectures.

HIVSIM produces several data output files. Some of these files allow the complete re-creation of the simulation state at the end of a run, while other files simulate the collection of data from individuals and their partnerships. The data collection files record person-specific and partnership-specific information. The person-specific file contains one data observation for each individual in the population with variables corresponding to the attributes associated with the individual, critical event times experienced by the individual during the simulation period, and summarized data regarding the partnerships and sexual behavior of the individual. The partnership-specific 
file contains one data observation for each partnership that occurred during the simulation run with the variables corresponding to characteristics of the individuals in the partnership, critical event times experienced during the partnership and detailed data regarding the sexual activity and HIV transmission experienced during the partnership. The combination of the information contained in these two files is used to compute all of the information required by the RPT study design. These two files are read into a statistical analysis software package, SAS V6.12 [53] and analyzed using SAS program code and macros developed to calculate the required vaccine efficacy estimates.

\section{Simulation Experiment Example}

\subsection{Vaccine Study Simulation Process Overview}

Prior to commencing a vaccine trial experiment, a partnership stabilization period must be run in order to allow a stable partnership contact pattern to develop. After the partnerships have been allowed to form, terminate and re-form for a prolonged period of time, the partnership contact patterns settle into a stable state consistent with the mixing propensities specified in the simulation parameters. Once this stabilization has occurred, all simulation repetitions for an experiment are started from the same initial partnership formation state. At the start of each simulation repetition, a number of individuals are randomly selected from the population and are infected. Immediately thereafter a simulated vaccine trial is initiated by:

1. The random selection of vaccine trial participants within the population, and

2. Their randomization to the vaccine or placebo arms of the vaccine trial.

Sexual mixing and the HIV transmission process continue throughout the simulated vaccine trial period, and at the end of the specified period the vaccine efficacy measurements from the trial are calculated and recorded. These steps involved in conducting a simulation experiment with HIVSIM are summarized below.

1. Run a partnership stabilization (i.e., burn-in ) period.

2. Introduce infection into the population.

3. Start the vaccine trial.

4. Resume sexual mixing (with HIV transmission and HIV vaccine effects).

5. End the vaccine trial.

6. Tabulate the estimates of $V E_{S}, V E_{I}$ and $V E_{R}$.

\subsection{Empirical Power Comparison between the RPT and Standard Trial Design}

A simulation experiment comparing the empirical power of the RPT design described in Section 3 and the standard vaccine design is provided as an example of how HIVSIM is used to evaluate the characteristics of the HIV vaccine trial designs. This example simulation experiment compares the statistical power of the RPT and the standard design when the vaccine effect on susceptibility is low and the vaccine effect on infectiousness is high.

One hundred repetitions of the simulated vaccine trials were conducted with $\mathrm{VE}_{\mathrm{S}}$ set to .25 and $\mathrm{VE}_{\mathrm{I}}$ set to .90. The simulated data from both the standard vaccine trial design and the RPT design were collected from each repetition in the simulation experiment. The experiment also included 200 repetitions of a vaccine trial where the vaccine had no effect $\left(\mathrm{VE}_{\mathrm{S}}=0, \mathrm{VE}_{\mathrm{I}}=0\right)$ in order to generate a null distribution for the purpose of making an empirical power comparison of the two study designs. Identical simulation parameters were used for all replications used in this experiment, with the exception of the null distribution runs where $V_{S}$ and $V E_{I}$ were set to zero. Some of the simulation parameter settings for this experiment are as follows:

- The vaccine trial study period was two years.

- The population consisted of 4,400 individuals.

- $75 \%$ of the population was monogamous and $25 \%$ of the population had a concurrency potential of two partnerships.

- The average partnership duration between pairs of monogamous partners was 145 days.

- The average time to seek out new partners for monogamous individuals was 60 days.

Table 1 provides the per-sex act transmission probabilities used in the experiment.

The simulated vaccine trials were conducted in a mixing population of 4,400 individuals. The individuals could either be exclusively monogamous or could have up to two concurrent sexual partners. After assignment of the individual sexual preferences and sexual activity traits, the partnership stabilization period was run until the contact matrices for these characteristics stabilized. Once stabilization was reached, a simulation start-up file was generated which allowed all of the repetitions used in this experiment to begin from the same mixing population partnership configuration. At the start of each simulation repetition, 400 individuals were randomly selected from the mixing population and infected with HIV. In order to

Table 1. RPT simulation experiment design transmission probability parameters

\begin{tabular}{|lcll|}
\hline $\begin{array}{c}\text { Per-Sex Act } \\
\text { Trans. Probs. }\end{array}$ & $\begin{array}{c}\text { Primary } \\
\text { Infection }\end{array}$ & $\begin{array}{c}\text { Clinical } \\
\text { Latency }\end{array}$ & $\begin{array}{c}\text { ARC/ } \\
\text { AIDS }\end{array}$ \\
\hline Anal Insertive & 0.25 & 0.125 & 0.05 \\
Anal Receptive & 0.0438 & 0.0022 & 0.0088 \\
Oral Insertive & 0.0063 & 0.0022 & 0.0088 \\
Oral Receptive & 0.0063 & 0.0003 & 0.0013 \\
\hline
\end{tabular}


simulate the process of conducting the vaccine trial in a population with a well-established HIV epidemic, the infected individuals were started at different stages of HIV infection; $8 \%$ of the population was started in the primary stage, $76 \%$ in the secondary stage and the remaining $16 \%$ was started in the ARC/ AIDS stage.

Following the introduction of HIV into the mixing population, 600 vaccine trial participants were enrolled and half were randomized to placebo, while the other half were randomized to vaccine. Sexual mixing and HIV transmission continued for a two-year vaccine trial period during each simulated repetition. At the end of the simulated vaccine trials, $\mathrm{VE}_{\mathrm{S}}$ was calculated for the standard vaccine trial design and $V E_{S}, V E_{I}$ and $V E_{R}$ were calculated for the RPT design. These steps are summarized below.

1. Set the vaccine trial study period to two years.

2. Randomly assign the number of potential concurrent sexual partners to one or two.

3. Randomly assign sexual preferences to one of \{Anal, Oral, Insertive, Receptive \}.

4. Randomly assign sex-act frequency to be either high or low.

5. Run a partnership stabilization period.

6. Introduce 400 infected individuals into the population of which $8 \%$ are in primary infection, $76 \%$ are in clinical latency and $16 \%$ have ARC/AIDS.
7. Start the HIV vaccine trial.

8. Enroll 600 mixing population members in the vaccine trial.

9. Randomize the vaccine trial members to placebo or vaccine.

10. Resume sexual mixing (with HIV transmission and HIV vaccine effects).

11. End the vaccine trial and estimate $V E_{S}, V E_{I}$ and $\mathrm{VE}_{\mathrm{R}}$.

\subsection{Simulation Experiment Results-Empirical Power Comparison between RPT and Standard Trial Designs}

The following information relevant to vaccine trial planning was obtained from the simulated vaccine trials. The average annual incidence in the simulated mixing population during the vaccine study period was $3.5 \%$. The average per-partnership transmission probability was $5 \%$. Within the vaccine study population, there were an average of 31 infected study trial subjects generated in each simulation repetition. During the two-year study period, each infected vaccine study participant had an average of 5.4 partnerships in which they could have possibly infected the other partner.

The relative statistical power of the standard vaccine trial design and the RPT design were compared. Figures 4 and 5 illustrate this comparison. The standard design is capable of producing estimates only for

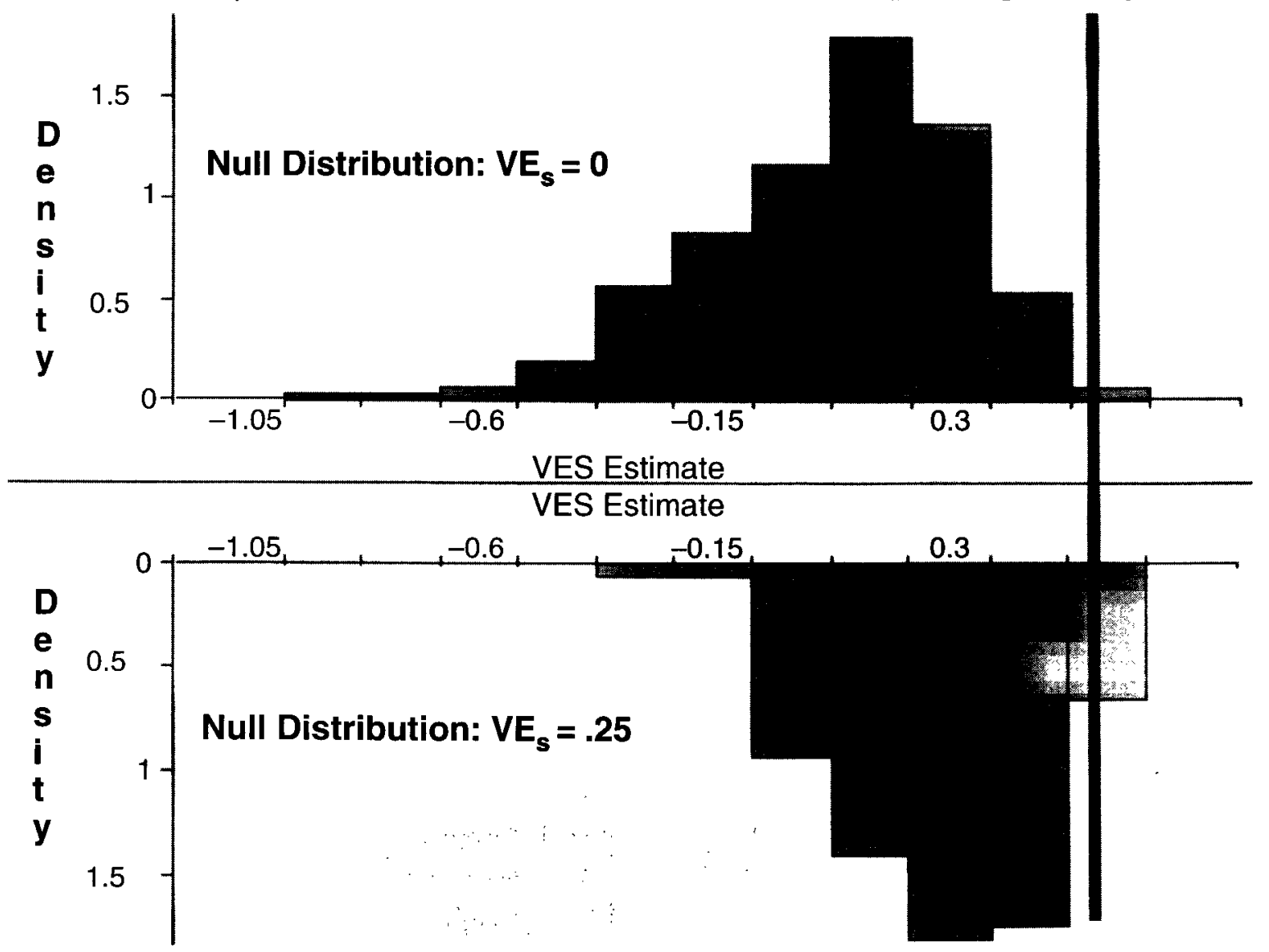

Figure 4. $\mathrm{VE}_{\mathrm{S}}$ empirical statistical power demonstration as calculated in both standard and RPT designs. 


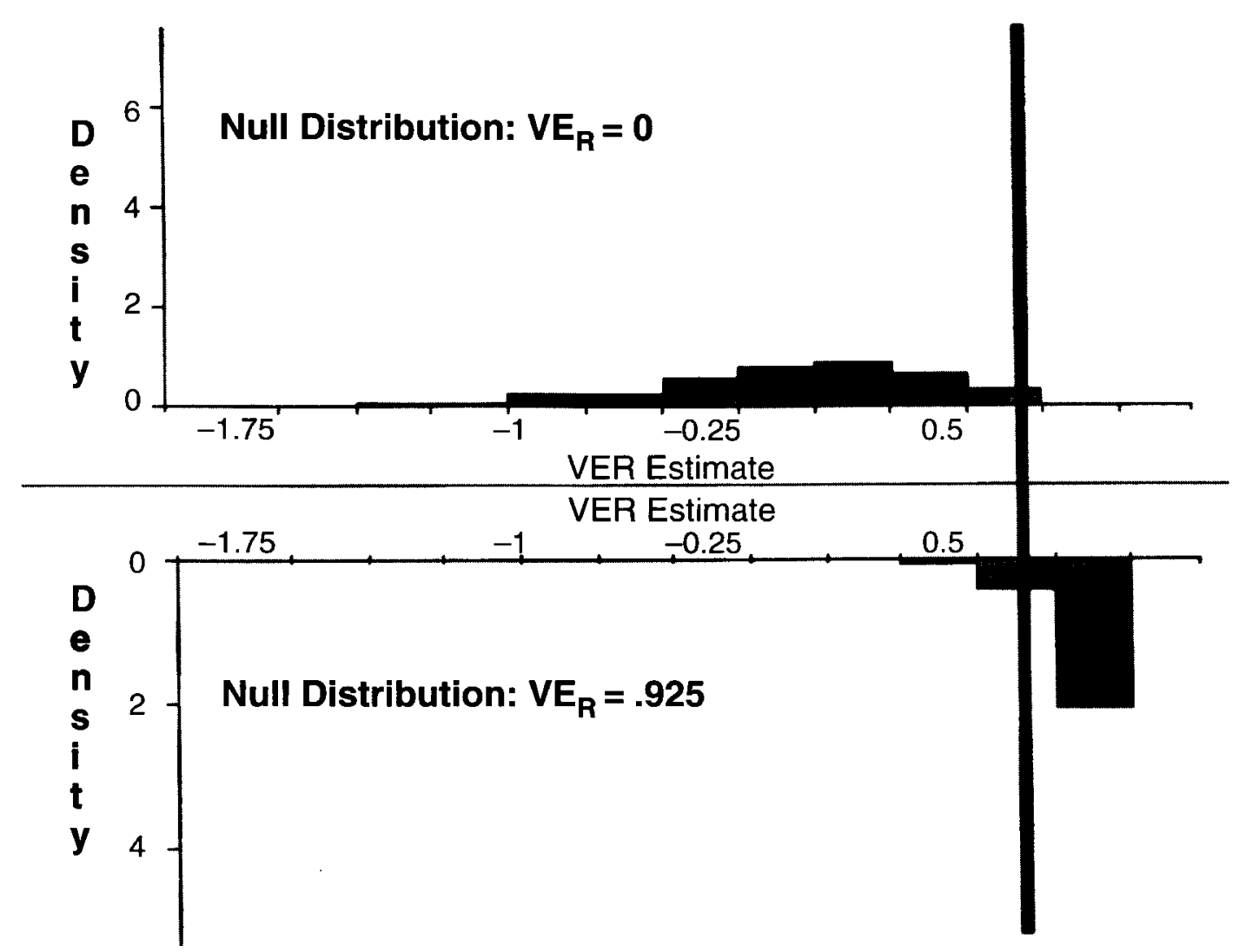

Figure 5. $V E_{\mathrm{R}}$ empirical power demonstration for the RPT design. Note that $\mathrm{VE}_{\mathrm{R}}$ cannot be calculated in the standard design.

$\mathrm{VE}_{\mathrm{S}}$. In Figure 4, the null distribution for $\mathrm{VE}_{\mathrm{S}}$ is presented on the same horizontal scale as the distribution of $V E_{S}$ when $V E_{S}$ was set to .25. As would occur in any real-world vaccine trials, the random events related to partnership formation and HIV transmission cause the realized $V_{S}$ estimates from each simulation to form a distribution of $\mathrm{VE}_{S}$ estimates centered on the actual vaccine effects possessed by the vaccines. Because of the low $\mathrm{VE}_{\mathrm{S}}$ in this case, the two distributions overlap considerably. The vertical line in Figure 4 indicates the rejection region on the null distribution for an alpha level of .05. The corresponding area to the right of this line on the $\mathrm{VE}_{\mathrm{S}}=.25$ distribution includes only $11 \%$ of the simulated vaccine trials, indicating that the statistical power would be particularly poor under these circumstances. The RPT design would obtain exactly the same estimate for $\mathrm{VE}_{\mathrm{S}}$ as realized by the standard trial design. In $89 \%$ of the simulated vaccine trials, both the standard design and the RPT design would have rejected the vaccine due to the poor $\mathrm{VE}_{\mathrm{S}}$ effects of the vaccine.

In contrast to the standard design, the RPT design is capable of producing estimates of $\mathrm{VE}_{\mathrm{I}}$ and $\mathrm{VE}_{\mathrm{R}}$. Figure 5 presents the distributions for $V_{R}$ obtained by the RPT design. In this case, there is very little overlap between the null distribution and the distribution of the $V_{R}$ as set by the simulation parameters $\left(\mathrm{VE}_{\mathrm{R}}=.925\right)$. The empirical power of the RPT design to detect the $V_{R}$ effect in this case was $95 \%$. Under the simulated conditions, the RPT design was clearly demonstrated to have more statistical power to detect the complete vaccine effects than the standard vaccine trial design. Use of the standard design under these circumstances would have frequently rejected vaccines which were capable of halting epidemic HIV transmission dynamics by reducing the basic reproduction number of the HIV epidemic to below one. The RPT design rarely rejected vaccines capable of halting epidemic transmission dynamics under these same circumstances because of its ability to measure infectiousness effects.

\section{Conclusions}

This paper described the development of a discreteevent simulation for the purpose of evaluating the RPT vaccine trial design and the associated statistical methods for calculating vaccine effects. The HIVSIM DES simulation program allowed detailed experiments to be simulated with different vaccine trial designs. Simulated data were collected and analyzed as they would have been under the proposed standard and RPT vaccine trial designs. The RPT design proposed here was demonstrated to have superior statistical power for vaccines with low susceptibility effects and strong infectiousness effects.

In addition, DES allowed the RPT design's statistical power to be quantified for situations exhibiting complex dynamics. It is not clear that this could be so readily accomplished using standard compartmental 
model analysis or Monte Carlo sampling of independent partnership and transmission events. This experiment demonstrates the utility of using DES to generate data for the evaluation of epidemiologic study designs and statistical methods in circumstances where complex transmission system dynamics are being modeled. Because this area is not well addressed in epidemiologic science at present, it is anticipated that the use of DES to generate data for the evaluation of infectious disease study designs and statistical methods could become a much more extensively utilized method in infectious disease epidemiology.

\section{References}

[1] Koopman, J. S., Simon, C. P., and Jacquez, J. A. "Assessing Contagiousness Effects of Vaccines and Risk Factors for Transmission." Modeling the AIDS Epidemic: Planning, Policy and Prediction, E. H. Kaplan and M. I. Brandeau, editors, Raven Press, New York, pp 439-460, 1994

[2] Koopman, J. S., Little, R. J. "Assessing HIV Vaccine Effects." American Journal of Epidemiology, Vol. 142, No.10, pp 11131120, 1995.

[3] Rida, W. N. "Assessing the Effect of HIV Vaccination on Infectiousness." Statistics in Medicine, Vol.15, No. 21-22, pp 2393-2404, 1996.

[4] Halloran, M. E. "Discussion: Assessing the Effect of HIV Vaccination on Infectiousness." Statistics in Medicine, Vol. 15, No. 21-22, pp 2405-2412, 1996

[5] Longini, I. M. Jr., Datta, S., Halloran, M. E. “Measuring Vaccine Efficacy for Both Susceptibility to Infection and Reduction in Infectiousness for Prophylactic HIV-1 Vaccines." Journal of Acquired Immune Deficiency Syndrome Human Retrovirology, Vol. 13, No. 5, pp 440-447, 1996.

[6] Halloran, M. E., Longini, I. M. Jr., Struchiner, C. J. "Study Designs for Evaluating Different Efficacy and Effectiveness Aspects of Vaccines." American Journal of Epidemiology, Vol. 146, No. 10, Nov. 15, pp 789-803, 1997

[7] Rida, W., Fast, P., Hoff, R., Fleming, T. "Intermediate-Size Trials for the Evaluation of HIV Vaccine Candidates: A Workshop Summary." Journal of Acquired Immune Deficiency Syndrome Human Retrovirology, Vol. 16, No. 3, pp 195-203, 1997

[8] Datta, S., Halloran, M. E., and Longini, I. M. Jr. “Augmented HIV Vaccine Trial Design for Estimating Reduction in Infectiousness and Protective Efficacy."Statistics in Medicine, Vol. 17, pp 185-200, 1998

[9] Longini, I. M. Jr., Sagatelian, K., Rida, W. N., Halloran, M. E. "Optimal Vaccine Trial Design when Estimating Vaccine Efficacy for Susceptibility and Infectiousness from Multiple Populations." Statistics in Medicine, Vol. 17, pp 1121-1136, 1998.

[10] Longini, I., Kitayaporn, D., Vanichseni, S., Choopanya, K., Hudgens, M., Halloran, M., Mastro, T., Mock, P. "Estimation of Vaccine Efficacy for Prophylactic HIV Vaccines from Field Trials in Developing Countries." International Conference on AIDS, Vol. 12, No. 1, pp 955 (abstract no. 435552), 1998.

[11] Smith, P. G., Rodrigues, L. C., Fine, P. E. "Assessment of the Protective Efficacy of Vaccines Against Common Diseases Using Case-Control and Cohort Studies." International Journal of Epidemiology, Vol. 13, No. 1, pp 87-93, 1984.

[12] Halloran, M. E., Struchiner, C. J., Longini, I. M. Jr. "Study Designs for Evaluating Different Efficacy and Effectiveness Aspects of Vaccines." American Journal of Epidemiology, Vol. 146, No. 10, pp 789-803, 1997.

[13] Rhodes, P. H., Halloran, M. E., and Longini, I. M. Jr. "Counting Process Models for Infectious Disease Data: Distinguishing Exposure to Infection from Susceptibility." Journal of Research Statistics, Soc. B. Vol. 58, No. 4, pp 751-762, 1996.

[14] Chen, R. T., Orenstein, W. A. "Epidemiologic Methods in Immunization Programs." Epidemiologic Reviews, Vol. 18, No. 2, pp 99-117, 1996
[15] Orenstein, W. A., Bernier, R. H., Hinman, A. R. “Assessing Vaccine Efficacy in the Field. Further Observations." Epidemiologic Reviews, Vol. 10, pp 212-41, 1988.

[16] Orenstein, W. A., Bernier, R. H., Dondero, T. J., Hinman, A. R., Marks, J. S., Bart, K. J., Sirotkin, B. "Field Evaluation of Vaccine Efficacy." Bulletin of the World Health Organization, Vol. 63, No. 6, pp 1055-68, 1985.

[17] Basch, P. F. Vaccines and World Health: Science, Policy, and Practice, Oxford University Press, New York, 1994.

[18] Longini, I. M., Hudgins, M., Halloran, M. E., Sagatelian, K. “A Markov Model for Measuring Vaccine Efficacy for Both Susceptibility to Infection and Reduction in Infectiousness for Prophylactic HIV Vaccines." Statistics in Medicine, in press, 1998.

[19] Datta, S., Halloran, M. E., Longini, I. M. Jr. "Augmented HIV Vaccine Trial Design for Estimating Reduction in Infectiousness and Protective Efficacy." Statistics in Medicine, Vol. 17, No. 2, pp 185-200, 1998.

[20] Longini, I. M. Jr., Datta, S., Halloran, M. E. “Measuring Vaccine Efficacy for Both Susceptibility to Infection and Reduction in Infectiousness for Prophylactic HIV-1 Vaccines." Joumal of Acquired Immune Deficiency Syndromes and Human Retrovirology, Vol. 13, No. 5, pp 440-7, 1996.

[21] Jacquez, J. A. Compartmental Analysis in Biology and Medicine, Third Edition, BioMedware, Ann Arbor, Michigan, 1996.

[22] Rhodes, P. H., Halloran, M. E., and Longini, I. M. Jr. "Counting Process Models for Infectious Disease Data: Distinguishing Exposure to Infection from Susceptibility." Journal of Research Statististics Soc. B, Vol. 58, No. 4, pp 751-762, 1996.

[23] Anderson, R. M., May, R. Infectious Diseases in Humans: Dynamics and Control, Oxford University Press, Oxford, 1991

[24] Blower, S. M. and McLean, A. R. "Prophylactic Vaccines, Risk Behavior Change and the Probability of Eradicating HIV in San Francisco." Science, Vol. 265, pp 1451-1454, 1994.

[25] Leitner, T., Escanilla, D., Franzen, C., Uhlen, M., Albert, J. “Accurate Reconstruction of a Known HIV-1 Transmission History by Phylogenetic Tree Analysis." Proceedings of the National Academy of Science USA, Vol. 93, No. 20, pp 10864$10869,1996$.

[26] Arnold, C., Balfe, P., Clewley, J. P. "Sequence Distances Between ENV Genes of HIV-1 from Individuals Infected from the Same Source: Implications for the Investigation of Possible Transmission Events."Virology, Vol. 211, No. 1, pp 198-203, 1995.

[27] Crandall, K. A. “Intraspecific Phylogenetics: Support for Dental Transmission of Human Immunodeficiency Virus." Journal of Virology, Vol. 69, No. 4, pp 2351-2356, 1995.

[28] Delwart, E., Busch, M., Kalish, M., Mosley, J., and Mullins, J. "Rapid Molecular Epidemiology of Human Immunology Virus Transmission." AIDS Research and Human Retroviruses, Vol. 11, No. 9, pp 1081-1093, 1995.

[29] Halloran, M. E. Concepts of Infectious Disease Epidemiology in Modern Epidemiology, Second Ed., K. J. Rothman and S. Greenland, editors, Lippincott-Raven, Philadelphia, 1998.

[30] Barth-Jones, D. C., Adams, A., Lange, K., Chick, S., Koopman, J. S. "Retrospective Partner Trials HIV Vaccine Study Design for Measurement of Vaccine Effect." Proceedings of the 12th World AIDS Conference, Prevention and Epidemiology, Geneva, Switzerland, Monduzzi Editore: Bologna, Italy, Vol. 3, pp 285-289, 1998.

[31] Barth-Jones, D., Adams, A., Lange, K., Koopman, J. “Retrospective Partner Trials HIV Vaccine Study Design for Measurement of Vaccine Effects." International Conference on AIDS, Vol. 12, No. 1. pp 954 (abstract no. 43549), 1998

[32] Dietz, K. "On the Transmission Dynamics of HIV." Math. Biosci., Vol. 90, pp 397-414, 1988.

[33] Dietz, K. and Hadeler, K. P. "Epidemiological Models for Sexually Transmitted Diseases." Journal of Math. Biol., Vol. 26, No. 1, pp 1-25, 1988

[34] Morris, M. "Sexual Networks and HIV." AIDS, Vol. 11, (Suppl A), pp S209-216, 1997.

[35] Mastro, T. D. and De Vincenzi, I. "Probabilities of Sexual HIV-1 Transmission." AIDS, Vol. 10 (Suppl A), No. S75-82, 1996.

[36] Leynaert, B., Downs, A. M., De Vincenzi, I. “Heterosexual Transmission of Human Immunodeficiency Virus: Variability 
of Infectivity Throughout the Course of Infection. European Study Group on Heterosexual Transmission of HIV." American Journal of Epidemiology, Vol. 148, No. 1, pp 88-96, 1998.

[37] Longini, I. M. Jr., Clark, W. S., Haber, M., and Horsburgh, R. Jr "The Stage of HIV Infection: Waiting Times and Infection Transmission Probabilities." Mathematical and Statistical Approaches to AIDS Epidemiology, C. Castillo-Chavez, editor, Springer-Verlag, Berlin, New York, Vol. 83, pp 111-137. Lecture Notes in Biomathematics, 1989.

[38] Jacquez, J. A., Koopman, J. S., Simon, C. P., and Longini, I. M. Jr. "Role of Primary Infection in Epidemic of HIV Infection in Gay Cohorts." Journal of Acquired Immune Deficiency Syndrome, Vol. 7, No. 11, pp 1169-1184, 1994.

[39] Koopman, J. S., Simon, C. P., Jacquez, J. A., and Park, T. S. "Selective Contact within Structured Mixing with an Application to HIV Transmission Risk from Oral and Anal Sex." Mathematical and Statistical Approaches to AIDS Epidemiology, C. Castillo-Chavez, editor, Springer-Verlag, Berlin, New York, 83, pp 316-348. Lecture Notes in Biomathematics, 1989.

[40] Ross, M. W., Rosser, B. R. "Dimensions of Sexual Behaviour in Homosexual Men: Replicability Across Time and Country." Psychol. Rep., Vol. 68, No. 2, pp 607-612, 1991.

[41] Coxon, A. P. M., Coxon, N. H., Weatherburn, P., et al. "Sex Role Separation in Sexual Diaries of Homosexual Men." AIDS, Vol. 7, pp 877-882, 1993.

[42] Van Druten, H., Van Griensven, F. and Hendricks, J. "Homosexual Role Separation: Implications for Analyzing and Modeling the Spread of HIV." Journal of Sexual Research, Vol. 29, pp 477-499, 1992.

[43] Morris, M., Kretzschmar, M. “Concurrent Partnerships and the Spread of HIV." AIDS, Vol. 11, No. 5, pp 641-648, 1997

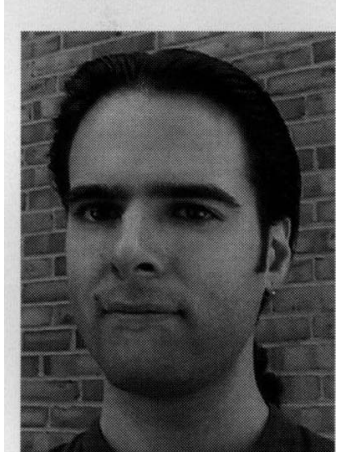

Andrew L. Adams is a Software Engineer at the University of Michigan Department of Epidemiology and at Merit Network, Inc., housed at the University of Michigan. He was the lead architect and developer for the HIVSIM model. Adams holds a BSE in Computer Engineering and an MSE in Computer Science, both from the University of Michigan. His research interests include stochastic and agent-based modeling and simulation, and Internet routing and management technologies.

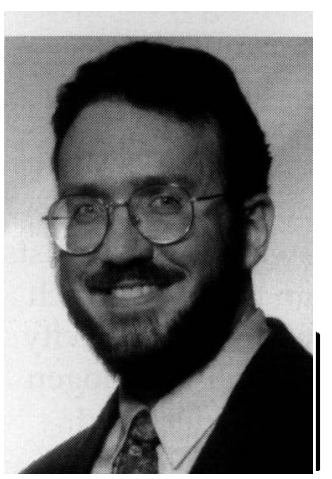

Daniel Barth-Jones is a doctoral candidate in Epidemiological Science at the University of Michigan, where he also received his Master of Public Health degree in General Epidemiology. Along with James Koopman, he developed the RPT design for HIV vaccine trials, which is his dissertation research topic. Barth-Jones' primary research interests include vaccinology, the epidemiology of HIV and sexually transmitted diseases, social network analysis and mathematical and computer simulation modeling of infectious diseases. He has worked as an epidemiologist/biostatistician in a variety of health care settings over the past 13 years. He presently has a consulting business which specializes in epidemiological and biostatistical consulting for managed care organizations.
[44] Morris, M. and Kretzschmar, M. "Concurrent Partnerships and Transmission Dynamics in Networks." Social Networks, Vol. 17, pp 299-318, 1995.

[45] Anderson, R. M. “The Epidemiology of HIV Infection: Variable Incubation Plus Infection Periods and Heterogeneity in Sexual Activity." J. R. Stat. Soc. A., Vol. 151, pp 66-93, 1988.

[46] Anderson, R. M., Gupta, S., and Ng, W. "The Significance of Sexual Partner Contact Networks for the Transmission Dynamics of HIV." Journal of Acquired Immune Deficit Syndrome, Vol. 3, pp 417-429, 1990.

[47] Morris, M., Zavisca, J., Dean, L. "Social and Sexual Networks Their Role in the Spread of HIV/AIDS Among Young Gay Men." AIDS Educ. Prev., Vol. 7, No. 5 (Suppl.), pp 24-35, 1995.

[48] Morris, M. "Sexual Networks and HIV." AIDS, Vol. 11 (Suppl A), pp 209-216, 1997

[49] Jacquez, J., Simon, C., and Koopman, J. "Structured Mixing: Heterogeneous Mixing by the Definition of Activity Groups." Mathematical and Statistical Approaches to AIDS Epidemiology, C. Castillo-Chavez, ed. Springer-Verlag, Berlin, New York, Vol. 83, pp 301-315, Lecture Notes in Biomath., 1989.

[50] Koopman, J., Simon, C., Jacquez, J., Joseph, J., Sattenspiel, L., Park, T. "Sexual Partner Selectiveness Effects on Homosexual HIV Transmission Dynamics." Journal of Acquired Immune Deficiency Syndrome, Vol. 1, No. 5, pp 486-504, 1988.

[51] Halloran, M. E., Longini, I. M. Jr., Struchiner, C. J. "Estimability and Interpretation of Vaccine Efficacy Using Frailty Mixing Models." American Journal of Epidemiology, Vol. 144, No. 1, pp 83-97, 1996.

[52] Longini, I. M. Jr. and Halloran, M. E. "A Frailty Mixture Model for Estimating Vaccine Efficacy." Applied Statistics, Vol. 45, No. 2, pp 165-173, 1996.

[53] SAS. SAS/Stat User's Guide, Ver. 6. Cary, NC, SAS Institute, 1992.

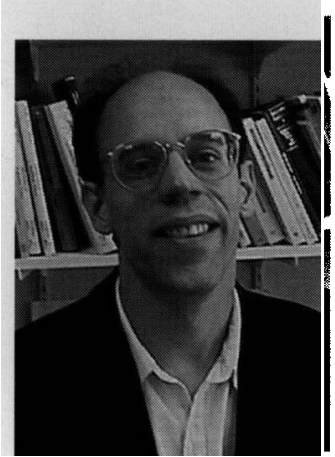

Stephen E. Chick is an Assistant Professor of Industrial and Operations Engineering at the University of Michigan. He received his PhD in 1995 from the Department of Industrial Engineering and Operations Research at the University of California at Berkeley. In addition to stochastic simulation, Chick's research interests include Bayesian statistics, decision analysis, computational methods in statistics, and applications in health care, particularly epidemiology. His work experience includes material-handling system design for the automotive industry using simulation analysis.

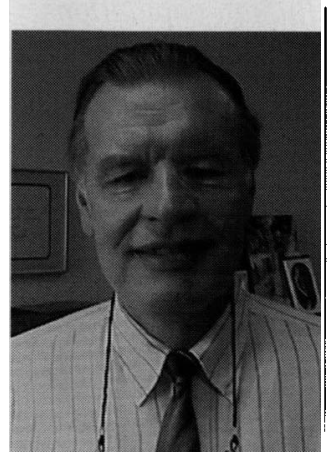

James Koopman is Professor of Epidemiology at the University of Michigan, where he received his medical degree. After a residency in pediatrics, he began his career in epidemiology as an epidemic intelligence service officer with the CDC. He received a Master's Degree in Public Health. He contributed to the effort to eradicate smallpox in India in 1973, and in 1984 instituted a stillongoing epidemiology program in Mexico. He now dedicates himself to developing a science of transmission system analysis. In this endeavor, Koopman helps organize public health department efforts to control infectious diseases, and designs and analyzes transmission system models. He views the science of transmission system analysis as pursuing divergent paths within the disciplines of applied mathematics and field epidemiology. 